\title{
Three-dimensional Numerical Study of a Kitchen Extractor Adopting Nearby Pumping Method
}

\author{
Jing Deng ${ }^{\mathrm{a}}$, Chongguang Hong ${ }^{\mathrm{b}}$, Guiquan Wang ${ }^{\mathrm{a}}$, Hongzhi Sheng ${ }^{\mathrm{a}}$ \\ anstitute of Mechanics, Chinese Academy of Sciences, Beijing 100190, China \\ ${ }^{b}$ Institute of Microelectronics, Chinese Academy of Sciences, Beijing 100190, China
}

Keywords: kitchen extractor; pumping mechanism; collection efficiency; numerical simulation; gas film cover

\begin{abstract}
A third generation kitchen extractor adopting nearby pumping method is investigated by using the computational fluid dynamics software. The numerical results are close to the experiment results, which can be used for supporting the optimum design of the equipment. The important parameters such as the particular distributions of velocity, temperature, and species fraction are obtained, which can supply more information about the pumping mechanism of this equipment. It is concluded that the gas-film-jet-cover technology significantly contributed to guaranteeing the pumping effect. Furthermore, decreasing negative pressure of pumping circle and/or the increasing positive pressure of gas-film-inlet could increase the collection efficiency of the equipment. However, it is necessary to choose the most appropriate pressure for getting enough collection efficiency with relatively low energy cost and working noise.
\end{abstract}

\section{Introduction}

Kitchen plays a very important role in the daily life. The air pollution is its main pollution which comes from the oil fumes generated in the cooking process, and it is especially severe in China due to the special cooking habits. The cooking oil fumes contain hundreds kinds of toxic substances, representatively including aldehydes, ketones, hydrocarbons and aromatic compounds [1], some of which have mutagenic and carcinogenic effects on human beings [2, 3]. Long-term exposure to cooking oil fumes may increase the risk for lung cancer. Most of the housewives are nonsmokers, however, lung cancer risk increasing with the number of meals per day to about threefold for housewives who cook meals everyday [4]. The risk is also greater if women usually wait until fumes are emitted from the cooking oil before they begin cooking (adjusted odds ratios=2.0-2.6) or they don't use a fume extractor (adjusted odds ratios=3.2-12.2) [5].

Nowadays, in order to reduce health hazards of cooking oil fumes to human beings, kitchen extractors are widely used to remove the cooking oil fumes. However, the pumping holes of the first generation kitchen extractor are above the pan, a lot of fumes in the kitchen have already been breathed in by people before they are pumped out. So the design needs to be improved. The pumping holes of the second generation kitchen extractor are on the wall beside the pan. It pumps the fumes to the opposite direction from people and looks better than the first generation. However, energy cost and working noise will also be increased when the second generation extractor is used with much higher pumping pressure. The third generation kitchen extractor has not been widely used. A typical example is the extractor adapting the nearby pumping method developed by Chongguang Hong et al. It uses an outstanding technology called 'gas-film-jet-cover', which form a planar jet between the positive pressure air inlet and the negative pressure pumping chamber. The difference of this design from others is that the pumping holes set in a circle around the pan, placed only $20 \mathrm{~mm}$ above the horizontal plane of the pan. Most of the fumes could be easily pumped out even under a smaller negative pressure. This equipment has been approved by government quality inspection department, which can achieve the collection efficiency as high as 99\% [6]. The third generation of kitchen extractor is as an alternative technology to the older ones, which also has other advantages such as much simpler structural components, lower working noise, lower energy cost, and easier maintenance. 
The collection efficiency of oil fumes is the key factor to test the pumping performance of the kitchen extractor. However, the experiment hardly be carried out to make detailed analysis of the flow field generated between the kitchen extractor and the pan. Researches on numerical simulation of new design for kitchen extractor of Wind-Curtain type [7], Inclined type [8], and the arrangement of kitchen ventilation [9] are already carried out. In order to carry out further design optimization of the third generation kitchen extractor, in this work, the extractor adopting nearby pumping method is investigated by using the computational fluid dynamics software, which is used to analyze influencing factors of the pumping mechanism and the collection efficiency.

\section{Model structure and numerical study method}

In this work, a comprehensive model of fluid dynamics and species transport is applied to the kitchen extractor modeling by using computational fluid dynamics commercial (CFD) software FLUENT. Pressure-Based solver in the FLUENT is chosen, because the low speed gas flow in the flow field of the kitchen extractor could be treated as incompressible. First of all, FLUENT solves conservation equations for mass and momentum. Although there are momentum and continuity equations for calculation of velocity and pressure, the continuity equation does not explicitly contain pressure. So at each iteration, a correction to the pressure distribution is calculated, designed to drive the local velocity field towards one that satisfies both momentum and continuity equations. The numerical coupling method that used in this work is called SIMPLE (Semi-Implicit Method for Pressure-Linked Equations) [10, 11]. Realizable k- $\varepsilon$ modeling is chosen for the turbulent modeling, which is suitable to simulate the weak turbulence and more accurately predicts the flow of both planar and swirl jets [11].

The equations used in this work are written in Table 1 , where $\vec{u}$ is the velocity vector, $T$ is the temperature, $p$ is the pressure, $\vec{\tau}$ is the stress tensor, $Y_{i}$ is the mass concentration of species $i, \vec{J}_{i}$ is the diffusion flux of species $i, \rho$ is the fluid density, $\mathrm{k}$ is the thermal conductivity, $\mathrm{g}$ is the gravitational acceleration, and $C p$ is the specific heat at constant pressure.

There are a few hundred $\mathrm{Pa}$ of the pressure variation and about $100 \mathrm{~K}$ temperature variation in the flow field, so the effect of the natural convection could not be ignored. In order to take into account the effect of buoyancy, the source term of the gravity in the momentum equation is considered. The FLUENT solver is used for implementing this case study based on the coupled calculation of equations in Table 1.

Table 1. CFD equiations

\begin{tabular}{llll}
\hline Equations & Convection term & Diffusion term & Source term \\
\hline Continuity & $\vec{u} \cdot \nabla \rho+\rho \nabla \cdot \vec{u}$ & 0 & 0 \\
Momentum & $\rho \vec{u} \cdot \nabla \vec{u}-\nabla p$ & $-\nabla \cdot \vec{\tau}$ & $\rho g$ in $y$ direction, 0 in $x, z$ directions \\
Energy & $\rho \vec{u} \cdot \nabla\left(C_{p} T\right)$ & $\nabla \cdot \nabla(k T)$ & 0 \\
Species & $\nabla \cdot\left(\rho \vec{u} Y_{i}\right)$ & $-\nabla \cdot \vec{J}_{i}$ & 0 \\
\hline
\end{tabular}

This work presents a three-dimensional, steady state numerical model to simulate the simplified real operation conditions. The geometric model of computational domain is illustrated in Fig. 1. The model is consisted by the side and the bottom of the pan, the pumping circle (a negative pressure generated by the pumping chamber), the gas film inlet, the gas inlet beside the pan (hot air generated by the natural gas stove) and the outside environment boundary. Meshing of the localmodel is shown in Fig. 2. Structured mesh is used in the area of outside environment. Tetrahedral mesh is generated in the area below the pumping circle. In order to improve the mesh quality and save computing time, the tetrahedral mesh is finally transformed into polyhedral mesh in the FLUENT solver. The total mesh number is about 2.4 million, which can ensure the calculation accuracy. 


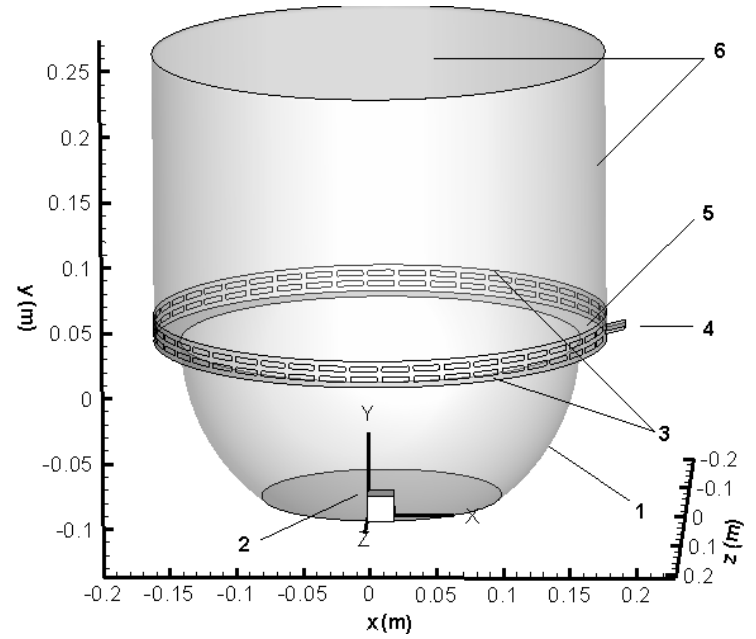

1-side of the pan; 2-bottom of the pan; 3pumping circle; 4-gas-film-jet inlet; 5-gas inlet beside the pan; 6-outside atmosphere

Fig. 1. Model structure of the kitchen extractor



Fig. 2. Meshing of the localmodel

In this model, as shown in Fig. 1, the bottom of the pan is treated as an inlet boundary, from where the mixed gas containing air (mole fraction of 95\%) and $\mathrm{H}_{2} \mathrm{O}$ stream (mole fraction of 5\%) with an atmospheric pressure and temperature of $400 \mathrm{~K}$ flow into the simulation area to simulate the cooking oil fumes. The concentrations of the stream, Oxygen and Nitrogen are calculated by species transport model. Collection efficiency of the kitchen ventilator is evaluated by the effect of the stream pumping.

The pumping circle is treated as a negative pressure outlet, from where the gas flowing into the calculation area is almost pumped out. The outside environment is treated as a pressure outlet with the total gauge pressure equals zero. The gas backflow with temperature of $300 \mathrm{~K}$ happens in the pressure outlet is allowed in FLUENT, so the gas can flow in and out of these boundaries due to the pressure-gradient.

In this work, the gas-film-jet with a temperature of $350 \mathrm{~K}$ produced by a positive pressure is a special design of the kitchen extractor, which has an incline angle with the horizontal plane and points to the inside of the pan. The gas-film-jet-cover technology could control the cooking oil fumes inside the pan effectively, which ensures the fumes are pumped out by the pumping circle as more as possible. The gas inlet beside the pan with a temperature of $350 \mathrm{~K}$ and total gauge pressure equals zero is used to simulate the hot gas generated by the gas stove.

Generally speaking, the pumping effect of this equipment is driven by the pressure gradient, on one hand, the pressure gradient generates between the pumping circle and the bottom of the pan, on the other hand, it generates between the pumping circle and the gas-film-jet. Negative pressure of the pumping circle and positive pressure of the gas-film-jet used in 10 simulation cases are presented in Table 2.

Table 2. Pressure boundary conditions in different cases

\begin{tabular}{|c|c|c|c|c|c|}
\hline Simulation conditions & Case1 & Case2 & Case3 & Case4 & Case5 \\
\hline Pumping circle negative pressure $(\mathrm{Pa})$ & -100 & -200 & -300 & -400 & -500 \\
\hline Gas-film-jet positive pressure $(\mathrm{Pa})$ & 200 & 200 & 200 & 200 & 200 \\
\hline Simulation conditions & Case6 & Case7 & Case8 & Case9 & Case10 \\
\hline Pumping circle negative pressure $(\mathrm{Pa})$ & -200 & -200 & -200 & -200 & -200 \\
\hline Gas-film-jet Positive pressure $(\mathrm{Pa})$ & 0 & 100 & 200 & 400 & 500 \\
\hline
\end{tabular}




\section{Simulation Results}

\section{D-calculation results of single case.}

Case 2 is a typical operation condition with the negative pressure of $-200 \mathrm{~Pa}$ and the positive pressure of $200 \mathrm{~Pa}$, which is the closest to the actual operation condition. Followings are the simulation results on the $\mathrm{z}=0$ sectional plane under the condition of Case2, as shown in Fig. 3.

Fig. 3(a) shows that the gas-film-jet-cover has a strong influence on the flow field, the horizontal surface above the pan is almost covered by the affected area, which ensures the gas controlled inside the pan. Fig. 3 shows that the maximum velocity of the gas-film-jet is about $18 \mathrm{~m} / \mathrm{s}$, which decreases along the jet direction, then increases before it flows into the pumping circle with the maximum value about $10 \mathrm{~m} / \mathrm{s}$. Furthermore, Fig. 3 also shows that the gas from the bottom of pan and the air from outside environment are pumped into the pumping circle by the entrainment of the gas-film-jet. Fig. 3(b) shows the mole fraction distribution of stream in the calculation area is decreasing along $y+$ direction, that's because the stream entering this area from the bottom of the pan is carried out continuously with the entrainment of gas-film-jet and the pumping effect of the pumping circle.



(a) Velocity and the path lines

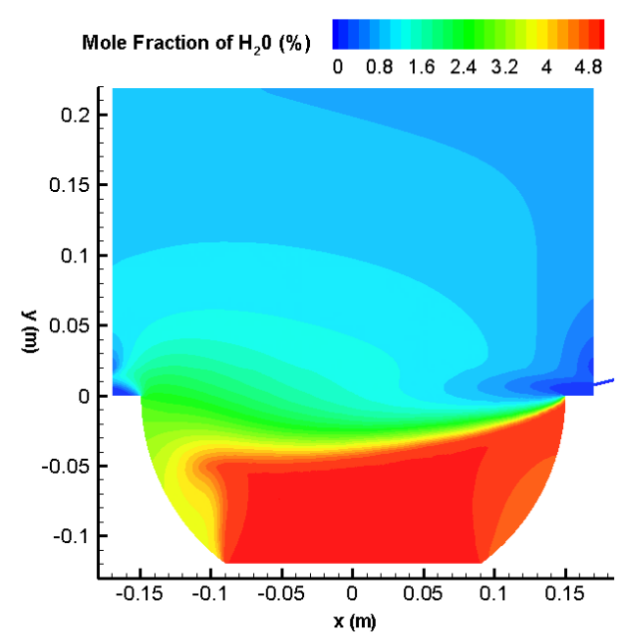

(b) Mole fraction of $\mathrm{H}_{2} \mathrm{O}$

Fig. 3. Calculation results of $\mathrm{z}=0$ sectional plane (Case 2)

\section{Comparison of modeling calculations with experimental results.}

Experiment measurement of flow velocity has been made by Chongguang Hong, et al. using the hot bulb-type anemometer which can obtain the magnitude of velocity in some important area. As shown in Fig. 4, the comparison of calculated velocity values in the vertical direction and the reverse direction of gas-film-jet of Case 7 with experimental results of $y=0.01 \mathrm{~m}$ sectional plan is given. Fig. 4(a) shows the calculations are very close to the experimental results. Meanwhile, Fig. 4(b) shows that the calculations are also very close to the experimental results on the left side in the opposite direction of gas-film-jet. However, the calculations are deviated from the experimental results on the right side due to the small difference of the structure between calculation model and experimental equipment. In general, the calculation results show agreement with the test results, which can be used as an important reference in further optimization of the kitchen extractor design. 


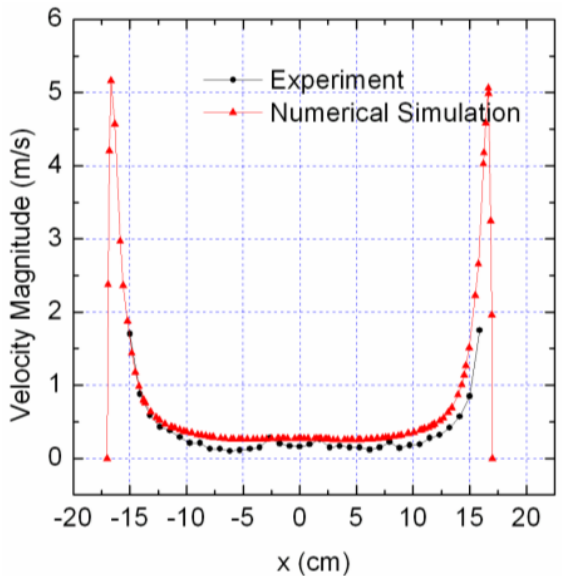

(a) The vertical direction of gas-film-jet



(b) The reverse direction of gasfilm-jet

Fig. 4. Comparison of calculations with experimental results of $y=0.01 \mathrm{~m}$ sectional plane

\section{Influence factors affecting the pumping efficiency}

According to the research of experiment and the numerical calculation, the main two influence factors affecting the pumping efficiency are concluded, one is the negative pressure generated by pumping circle, and another one is the positive pressure of the gas-film-jet. Followings are detailed analysis of the two main factors.

\section{Pumping pressure generated by pumping circle}

As shown in Table 2, in Case1-5, the negative pressure generated by the pump circle decreasing from $-100 \mathrm{~Pa}$ to $-500 \mathrm{~Pa}$ with the positive pressure of the gas-film-jet fixed to $200 \mathrm{~Pa}$.

Fig. 5 shows that the mole fraction distributions of stream along y+ direction. On one hand, the mole fraction of stream is decreasing with the decreasing pumping pressure, which indicates the collection efficiency is increasing with the decreasing pumping pressure. On the other hand, most of the stream is pumped out before arriving to the pump circle, and the minority of stream passed the pump circle may flow back and be pumped out, which also can be seen in Fig. 3(b).

Moreover, there is no obvious effect on collection efficiency with the decreasing negative pumping pressure from $-300 \mathrm{~Pa}$ (Case3-5). So the negative pressure should not be blindly decreased due to the consideration of energy cost and working noise.

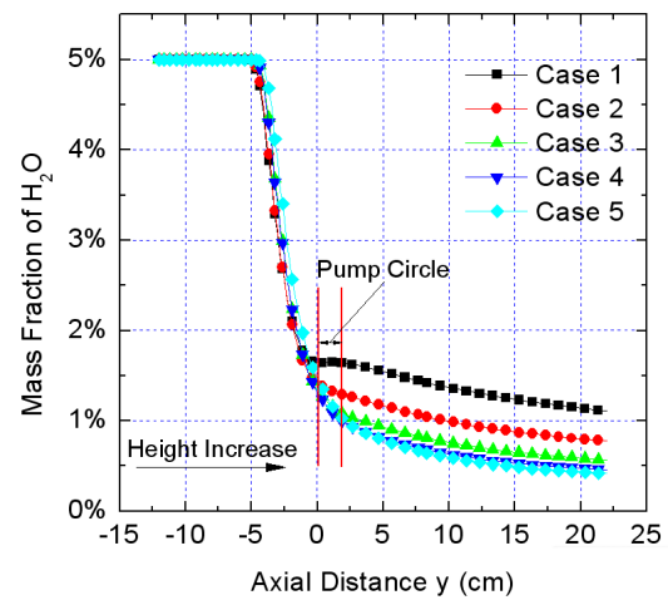

Fig. 5. Mole fraction of $\mathrm{H}_{2} \mathrm{O}$ along the central axis under different pumping pressure (Case1-5)

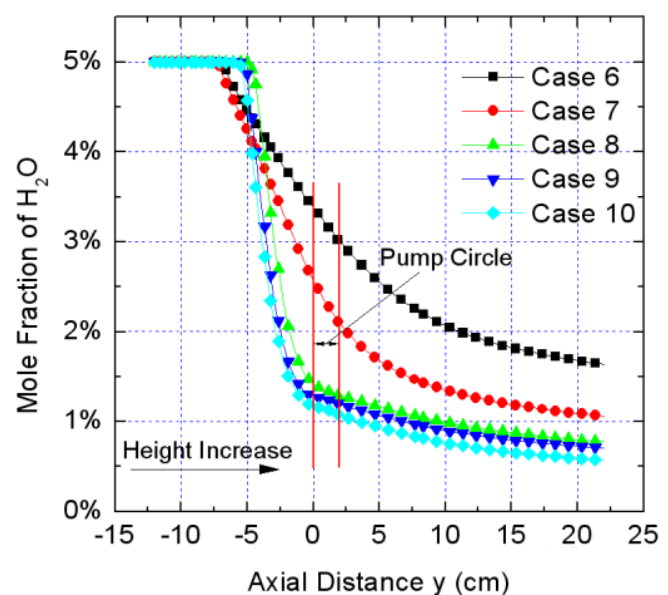

Fig. 6. Mole fraction of $\mathrm{H}_{2} \mathrm{O}$ along the central axis under different pressure of gas-film-jet (Case6-10) 


\section{Pressure of gas-film-jet}

As shown in Table 2, in Case6-10, the positive pressure of gas-film-jet is increasing from 0Pa to $500 \mathrm{~Pa}$ with the negative pressure generated by pumping circle fixed to $-200 \mathrm{~Pa}$.

Fig. 6 shows that the mole fraction distributions of stream along $y+$ direction, from where can be seen that no gas-film is generated in Case6, the mole fraction of stream is decreasing with the increasing height, but once the gas-film-jet-cover is used such as in Case 7 which even has only a positive pressure of $100 \mathrm{~Pa}$, there would be substantial increasing of the decreasing rate of the mole fraction of stream. When the positive pressure is increasing to $200 \mathrm{~Pa}$ such as in Case8, there is still a substantial increasing rate of collection efficiency. However, no obvious increasing rate with the increasing pressure can be seen when it is above 200Pa (Case8-10). Meanwhile, the excessive pursuit of increasing pressure may cause the waste of electricity and produce annoying working noise, which has been observed from the experiments.

\section{Conclusions}

In this work, a third generation kitchen extractor adopting nearby pumping method is investigated by using the computational fluid dynamics software FLUENT. The numerical results are close to the experiment results, which have great significance in the optimization design to the kitchen extractor.

The important parameters including the distributions of velocity and species fraction are obtained, which could supply more information about the pumping mechanism of this equipment. These numerical results prove that the gas-film-jet-cover has a very significant impact on the collection efficiency. The negative pressure outlet generated by the pump circle and the positive pressure generated by the inlet of the gas-film-jet should be coordinated, which can drive oil fumes flow into the exhaust channel effectively.

By using the stream to simulate the cooking oil fumes, and making a specific analysis of the collection efficiency in different 10 Cases, it is concluded that decreasing the pressure of the pumping circle and increasing the pressure of the gas-film-jet can increase the collection efficiency. However, excessively increasing positive pressure of gas-film-jet, and excessively decreasing negative pumping pressure generated by pump circle may cause the waste of electricity and produce annoying working noise.

\section{References}

[1] H. Chen, S. H. Ye. Effects on human health of cooking oilsmoke. Shanghai Environmental Sciences 1991;10:48-53.

[2] Y. M. Hou. Effects on human health of edible oil smoke. Shanghai Environmental Sciences. 1997; 16:35-36.

[3] S. G. Li, D. H. Pan. Analysis of polycyclic aromatic hydrocarbons in the edible oils and its heated products of some catering trades. Journal of Environment and Health. 1992;9:217-219.

[4] Y. T. Gao, W. Zheng, R. Zhang, R. N. Gao, C. W. Xu, M. L. Liao, Z. P. Yang, Z. Q. Lin, Z. X. Wang, J. J. Zhao, Y. S. Zhang, W. J. Blot, J. F. Fraumeni Jr. Contrast study of 627 female patients of Shanghai urban district. Oncology. 1986;7: 194-197.

[5] Y. C. Ko, L. S. C. Cheng, C. H. Lee, J. J. Huang, M. S. Huang, E. L. Kao, H. Z. Wang, H. J. Lin. Chinese food cooking and lung cancer in women nonsmokers. Am. J. Epidemiol. 2000;151:140-147.

[6] C. G. Hong. Application of pumping gas and dust by using fluid film technology in the household kitchen. China Housing Facilities. 2004;9:45-47

[7] M. X. Shi, H. Q. Wang, Z. Y. Wang, Y. Chen. Experimental research on new design for windcurtain lampblack presser. Journal of Hunan University of Technology. 2008;22:20-23.

[8] Y. Z. Zhu. Analysis of oilsmoke and pumping mechanism: part I. Household Appliance Technology. 1997;2:9-10.

[9] J. Yang, W. B. Liu. Numerical simulation of kitchen ventilation. Refrigeration \& AirConditioning. 2007;2:91-93.

[10]W. Q. Tao. Numerical Heat Transfer. 2nd ed. Xi'an: Xi'an Jiaotong University Press; 2001.

[11]ANSYS Inc. ANSYS FLUENT 12.0 Theory Guide . Lebanon, NH; 2009. 
Material Sciences and Manufacturing Technology

10.4028/www.scientific.net/AMR.629

Three-Dimensional Numerical Study of a Kitchen Extractor Adopting Nearby Pumping Method 10.4028/www.scientific.net/AMR.629.495 